DAMTP 96-71

SWAT $/ 126$

\title{
GENERIC CURRENT-CARRYING STRINGS
}

\author{
Anne-Christine Davis ${ }^{1}$ \\ Warren B. Perkins ${ }^{2}$ \\ 1. Department of Applied Mathematics and Theoretical Physics, \\ Silver Street, Cambridge, CB3 9EW, Great Britain \\ 2. Department of Physics, University of Wales Swansea, \\ Singleton Park, Swansea, SA2 8PP
}

\begin{abstract}
We investigate the standard model in a cosmic string background and show that the electroweak symmetry is partially restored. For a range of parameters the electroweak Higgs field can wind in this region, producing an electroweak string stabilised by the cosmic string background. In this case there are quark and lepton zero modes that result in the string becoming superconducting at the electroweak scale. If the electroweak Higgs field does not wind, there are no zero modes, but there are fermion bound states. These bound states can also carry a current.
\end{abstract}




\section{1) Introduction}

Topological defects, and in particular cosmic strings, are likely to arise as a result of phase transitions in the early universe. A network of such strings could explain the observed large scale structure and the anisotropy in the microwave background radiation [1]. They arise in many Grand Unified Theories (GUT) of particle physics and have high predictive power, the only free parameter being the mass per unit length. Recently, the possibility of superconducting strings [2], or current carrying strings in general, has received a revival of interest. This has been sparked by the realisation that current carrying strings may be more general than previously thought [3]. The cosmology of such strings could be modified considerably, see [4] and references therein. Hence, it is important to ascertain whether or not strings carry a current, and how large the current is.

Cosmic string superconductivity can arise as a result of boson condensates, fermion zero modes [2], or gauge boson condensates [5]. Strings can also carry a current as a result of fermion bound states [6]. The maximal current can be enormous, approaching the grand unified scale for GUT strings. Models containing strings carrying such enormous currents can be ruled out as they produce stable remnants, vortons [7], which would dominate the energy density of the universe. However, it was pointed out that strings could become current carrying at a subsequent phase transition [3] as a result of the restoration of, say, the electroweak symmetry around the string. In this case the resulting current would be proportional to the electroweak scale, it would produce only a modest change to the standard string cosmology, but could still change the microphysics of the string significantly.

In this paper we address this issue. We show that couplings between the string and the electroweak sector lead to at least partial restoration of the electroweak symmetry around the string. Such couplings arise either as direct couplings given by the GUT, or as effective couplings at higher order. Depending on the ratio of the couplings, as given by the GUT, the Higgs field can wind. If it does wind then there are zero modes of the ordinary quarks and leptons which give rise to fermion superconductivity. If the Higgs field doesn't wind then there are no zero modes, but there are bound states of the ordinary quarks and leptons. This is because there is an effective two dimensional potential, which always has at least one bound state. These bound states can carry a current [6]. Consequently, we conclude that a cosmic string can always carry a current, though the current may not be enormous, or persistent.

\section{2) The Electroweak $\times U(1)$ Model}

We work with a model in which we augment the Weinberg-Salam model with a string forming $\mathrm{U}(1)$ scalar field $\tilde{\chi}$ and associated gauge field $X_{\mu}$ [8]. The Lagrangian we consider is

$$
\begin{aligned}
\mathcal{L}= & \left(\tilde{D}_{\mu} \tilde{\chi}\right)^{\dagger}\left(\tilde{D}^{\mu} \tilde{\chi}\right)-\frac{1}{4} X_{\mu \nu} X^{\mu \nu}-\tilde{\lambda}\left(\tilde{\chi}^{\dagger} \tilde{\chi}-\tilde{\eta}^{2}\right)^{2}-\xi M^{2}\left(\tilde{\chi}^{\dagger} \tilde{\chi}-\tilde{\eta}^{2}\right) \Phi^{\dagger} \Phi \\
& +\left(D_{\mu} \Phi\right)^{\dagger}\left(D^{\mu} \Phi\right)-\frac{1}{4} Y_{\mu \nu} Y^{\mu \nu}-\frac{1}{4} W_{\mu \nu}^{a} W^{a \mu \nu}-\lambda\left(\Phi^{\dagger} \Phi-\eta^{2}\right)^{2},
\end{aligned}
$$

where the $\tilde{\chi}$ covariant derivative is

$$
\tilde{D}_{\mu}=\partial_{\mu}-i q X_{\mu}
$$


and the electroweak Higgs field has a charge $\alpha$ under the extra U(1) so that its covariant derivative is

$$
D_{\mu}=\partial_{\mu}-i \alpha X_{\mu}-\frac{i}{2} g \tau . \mathbf{W}_{\mu}-\frac{i}{2} g^{\prime} Y_{\mu} .
$$

We have also introduced a scalar-scalar cross coupling with magnitude $M^{2}$ and sign $\xi= \pm 1$. These couplings between the string and electroweak sectors are expected to arise generically either directly from the underlying GUT or as effective couplings at higher order.

We can write the quartic scalar terms in the Lagrangian in the form,

$$
V_{4}=\left(\begin{array}{cc}
\tilde{\chi}^{\dagger} \tilde{\chi} & \Phi^{\dagger} \Phi
\end{array}\right)\left(\begin{array}{cc}
\tilde{\lambda} & \xi M^{2} / 2 \\
\xi M^{2} / 2 & \lambda
\end{array}\right)\left(\begin{array}{c}
\tilde{\chi}^{\dagger} \tilde{\chi} \\
\Phi^{\dagger} \Phi
\end{array}\right)
$$

$V_{4}$ is unbounded below if the matrix has a negative eigenvalue, requiring that this is not the case yields the constraint

$$
M^{2} / 2<\sqrt{\tilde{\lambda} \lambda}
$$

Thus there is a natural limit to the magnitude of the cross coupling.

When the extra $U(1)$ symmetry is broken a string forms with the usual profile for the Higgs and gauge fields;

$$
\begin{gathered}
\tilde{\chi} \sim \mathrm{e}^{i \theta} \begin{cases}a r & \text { small } r \\
\tilde{\eta} & \text { large } r\end{cases} \\
X_{\theta} \sim \begin{cases}b r & \text { small } r \\
1 / q r & \text { large } r\end{cases}
\end{gathered}
$$

with the other components of $X_{\mu}$ being zero as usual. For concreteness we will call the energy scale of the string the GUT scale, but our analysis does not depend on the energy scale of string formation.

\section{3) The Electroweak Background Fields}

We can now consider the electroweak gauge and Higgs field profiles around the string. Whilst this was done in [8], the Higgs-Higgs interaction was neglected and a more general analysis is necessary. We work in temporal gauge and look for static solutions of the equations of motion. We can consistently set most of the electroweak fields to zero and look for solutions with only $Z_{\mu}\left(Z_{\mu}=-\frac{g}{G} W_{\mu}^{3}+\frac{g^{\prime}}{G} Y_{\mu}, G^{2}=g^{2}+g^{\prime 2}\right)$ and the lower component of the Higgs doublet, $\phi$, non-vanishing. The Lagrangian then reduces to

$$
\mathcal{L}=-\phi_{, r}^{2}-\left(\frac{G}{2} Z_{\theta}+\alpha X_{\theta}\right)^{2} \phi^{2}-\frac{1}{2}\left(Z_{\theta, r}+Z_{\theta} / r\right)^{2}-\lambda\left(\phi^{2}-\eta^{2}\right)^{2}-\xi \tilde{M}^{2}\left(\chi^{\dagger} \chi-1\right) \phi^{2}
$$

where $\tilde{\chi}=\tilde{\eta} \chi$ and $M^{2} \tilde{\eta}^{2}=\tilde{M}^{2}$. motion,

Applying the scalings: $\phi=\frac{\tilde{M}}{\sqrt{\lambda}} \psi, r=x / \tilde{M}$ and $G Z_{\theta} / \tilde{M}=z$ leads to the equations of

$$
\begin{gathered}
\frac{1}{x} \frac{d}{d x}\left(x \psi_{, x}\right)=\left[\left(\frac{z}{2}+\frac{\alpha}{\tilde{M}} X_{\theta}\right)^{2}-2 \eta^{2} \frac{\lambda}{\tilde{M}^{2}}+\xi\left(\chi^{\dagger} \chi-1\right)\right] \psi+2 \psi^{3} \\
\frac{1}{x} \frac{d}{d x}\left(x\left(z_{, x}\right)\right)=\frac{z}{x^{2}}+\frac{G^{2}}{\lambda}\left(\frac{z}{2}+\frac{\alpha}{\tilde{M}} X_{\theta}\right) \psi^{2}
\end{gathered}
$$


We have solved these equations numerically for a range of parameters. Some typical profiles are presented in figures 1-3. The important parameters in the model are the ratio of the new $\mathrm{U}(1)$ charges of the GUT Higgs and electroweak Higgs, $\frac{\alpha}{q}$, the Higgs-Higgs cross coupling, $M$, and the ratio of the electroweak energy scale to the GUT scale. In fig. 1 we show the variation with the Higgs-Higgs coupling, M; fig.2 shows the variation with the ratio of the gauge couplings $\alpha / q$; fig.3 shows the variation with the ratio of the two length scales. The corresponding $\mathrm{Z}$ profiles are shown in fig.4. The solid line corresponds to the same parameter values in all four figures and only the specified parameter is varied in each case.

The gauge coupling gives a positive contribution to the electroweak Higgs field mass term and the Higgs-Higgs cross coupling gives a contribution whose sign is determined by $\xi$. The latter contribution is restricted to the GUT string core, while the former varies as $1 / r^{2}$ outside the core. In fig. 1 we see the effects of the scalar cross coupling at small distances, inside the GUT string core the scalar cross coupling increases the Higgs expectation value, while outside the Higgs VEV is reduced due to the interaction with the string's gauge field. As the GUT length scale gets smaller relative to the electroweak scale the effects of the gauge coupling become dominant and the electroweak Higgs field is pushed towards zero at the core of the string. If the $1 / r$ form of the GUT gauge field were to persist into the core of the string, the electroweak Higgs field would be forced exactly to zero at the centre of the string as the only regular solution of the equation of motion is $\phi \propto r^{\alpha / q}$. This limit is considered in ref.9. The onset of this behaviour is displayed in fig 3 . If we attempt to construct a power series solution in the region $m_{E W} r<1, m_{G U T} r>1$ the string gauge field varies as $1 / r$ and the Higgs field varies as $r^{ \pm \alpha / q}$. As $r_{G U T}$ gets smaller the electroweak Higgs field at the centre of the string must drop to zero so as not to overshoot its asymptotic value. Thus as the GUT mass scale gets larger $\psi(0)$ tends to zero. As the $1 / r$ form of the gauge field does not persist to $r=0$, the Higgs field must have zero gradient at the origin, but the departure from the $r^{\alpha / q}$ form is restricted to the region $r<r_{G U T}$.

The profiles shown confirm our previous results [8] where a region of electroweak symmetry restoration of order the electroweak scale was found by considering the gauge coupling alone. We have a 'composite string'; the GUT string core is surrounded by a region of partially restored electroweak symmetry containing a $\mathrm{Z}$ magnetic flux.

If we introduce a winding into the electroweak Higgs field, i.e. $\phi=\phi \mathrm{e}^{i l \theta}$, then $\alpha X_{\theta}$ is simply replaced by $\alpha X_{\theta}+l / r$. For $r>r_{G U T}$ the ratio of gauge couplings, $\frac{\alpha}{q}$ is replaced by $\frac{\alpha}{q}+l$ while as $r \rightarrow 0$ we have a persistent $1 / r$ behaviour that forces $\psi(0)=0$ exactly. This change of boundary condition has little effect for $m_{G U T} \gg m_{E W}$, as discussed above, and the winding merely introduces an effective gauge coupling. The energy of the electroweak fields around the string increases as the gauge coupling ratio, $\frac{\alpha}{q}$, increases as shown in fig.5. For $g>0.5$ we expect the electroweak field energy to be minimised by introducing a winding in the electroweak Higgs field. This is consistent with our previous results [8]. In this case we really have an electroweak string surrounding the GUT core. The difference here is that the electroweak string is stabilised by the presence of the GUT core. The appearance of such Higgs field windings is considered in ref.9 in terms of the 'Cinderella' process seen in the pure electroweak string[10]. 


\section{4) Fermionic States in the String Background}

In this section we analyse the Dirac spectrum in the background of our string. We assume that the string has the most favourable winding and work in a gauge where there is no $\mathrm{W}$ condensate and the only non-zero electroweak fields are $\phi$ and $Z_{\theta}$. First we consider the case where the electroweak Higgs field has a non-trivial winding and search for fermion zero modes. We consider the non-winding case later in this section and search for fermion bound states.

In the background of the composite string the quark Lagrangian is

$$
\begin{aligned}
& \mathcal{L}_{\text {quark }}= \\
& \bar{u}_{L} \gamma^{\mu}\left(-i \partial_{\mu}-\frac{1}{2 G}\left(g^{2}-g^{\prime 2} / 3\right) Z_{\mu}+\nu X_{\mu}\right) u_{L}+\bar{d}_{L} \gamma^{\mu}\left(-i \partial_{\mu}+\frac{1}{2 G}\left(g^{2}+g^{\prime 2} / 3\right) Z_{\mu}+\nu X_{\mu}\right) d_{L} \\
& +\bar{u}_{R} \gamma^{\mu}\left(-i \partial_{\mu}+\frac{2}{3} \frac{g^{\prime 2}}{G} Z_{\mu}+\nu^{\prime} X_{\mu}\right) u_{R}+\bar{d}_{R} \gamma^{\mu}\left(-i \partial_{\mu}-\frac{1}{3} \frac{g^{\prime 2}}{G} Z_{\mu}+\tilde{\nu} X_{\mu}\right) d_{R} \\
& -G_{d}\left(\bar{d}_{L} \phi d_{R}+\bar{d}_{R} \phi^{*} d_{L}\right)+G_{u}\left(\bar{u}_{L} \phi^{*} u_{R}+\bar{u}_{R} \phi u_{L}\right) .
\end{aligned}
$$

where we have introduced couplings $\nu, \nu^{\prime}$ and $\tilde{\nu}$ between the quarks and the $X$ field. Gauge invariance requires

$$
\tilde{\nu}=\nu+\alpha, \quad \nu^{\prime}=\nu-\alpha
$$

where $\alpha$ is the charge of the electroweak Higgs field under the extra U(1) introduced in section 2. The generic form of the Lagrangian for each pair of particles is thus

$$
\mathcal{L}_{\mathrm{f}}=\bar{f}_{L} \gamma^{\mu}\left(-i \partial_{\mu}-a Z_{\mu}+c X_{\mu}\right) f_{L}+\bar{f}_{R} \gamma^{\mu}\left(-i \partial_{\mu}-b Z_{\mu}+d X_{\mu}\right) f_{R}+m\left(\bar{f}_{L} \phi^{*} f_{R}+\bar{f}_{R} \phi f_{L}\right)
$$

where $\phi$ should be replaced by $\phi^{*}$ in the case of the $d$ quark and electron. The Dirac equations are then

$$
\begin{gathered}
\gamma^{\mu}\left(-i \partial_{\mu}-a Z_{\mu}+c X_{\mu}\right) f_{L}+m \phi^{*} f_{R}=0 \\
\gamma^{\mu}\left(-i \partial_{\mu}-b Z_{\mu}+d X_{\mu}\right) f_{R}+m \phi f_{L}=0
\end{gathered}
$$

Using the Dirac matrices of ref.11 we have the following form for the left and right-handed spinors

$$
f_{L}=\left(\begin{array}{c}
f_{1} \\
f_{2} \\
-f_{1} \\
-f_{2}
\end{array}\right) \quad f_{R}=\left(\begin{array}{c}
f_{3} \\
f_{4} \\
f_{3} \\
f_{4}
\end{array}\right)
$$

Making the ansatz

$$
\left(\begin{array}{l}
f_{1} \\
f_{2} \\
f_{3} \\
f_{4}
\end{array}\right)=\mathrm{e}^{i w t-i k z+i n \theta}\left(\begin{array}{c}
A \\
B \mathrm{e}^{i \theta} \\
C \mathrm{e}^{i l \theta} \\
D \mathrm{e}^{(l+1) i \theta}
\end{array}\right), \quad \phi=\mathrm{e}^{i l \theta} \phi(r)
$$


the Dirac equations reduce to

$$
\left(\begin{array}{cccc}
w+k & -i\left(\begin{array}{c}
\partial_{r}+\frac{n+1}{r} \\
-a Z_{\theta}+c X_{\theta}
\end{array}\right) & m|\phi| & 0 \\
-i\left(\begin{array}{c}
-\partial_{r}+\frac{n}{r} \\
-a Z_{\theta}+c X_{\theta}
\end{array}\right) & -w+k & 0 & m|\phi| \\
-m|\phi| & 0 & -w+k & -i\left(\begin{array}{c}
-\partial_{r}-\frac{n+l+1}{r} \\
+b Z_{\theta}-d X_{\theta}
\end{array}\right) \\
0 & -m|\phi| & -i\left(\begin{array}{c}
\partial_{r}-\frac{n+l}{r} \\
+b Z_{\theta}-d X_{\theta}
\end{array}\right) & w+k
\end{array}\right)\left(\begin{array}{c}
A \\
B \\
C \\
D
\end{array}\right)=0
$$

This is now in the form where the standard zero mode analysis [12] can now be applied. Let $w=k$ and set $A=D=0$, at small distances the leading order terms in the Dirac equations reduce to

$$
\begin{aligned}
& -i\left(\partial_{r}+\frac{n+1}{r}\right) B+m|\phi| C=0 \\
& -m|\phi| B-i\left(\partial_{r}-\frac{n+l}{r}\right) C=0 .
\end{aligned}
$$

At small distances $\phi \propto r^{|l|}$ and we have two short distance solutions:

$$
\left(\begin{array}{c}
B \\
C
\end{array}\right) \sim\left(\begin{array}{c}
r^{n+l+|l|+1}+\ldots \\
r^{n+l}+\ldots
\end{array}\right)
$$

and

$$
\left(\begin{array}{c}
B \\
C
\end{array}\right) \sim\left(\begin{array}{c}
r^{-n-1}+\ldots \\
r^{-n+|l|}+\ldots
\end{array}\right) .
$$

Thus we have two regular small $r$ solutions for $n=-1,-2, . .,-l$; we have $|l|$ zero modes for $l$ positive.

Similarly for $w=-k$ we set $B=C=0$ and the small distance leading order terms in the Dirac equations reduce to

$$
-i\left(-\partial_{r}+\frac{n}{r}\right) A+m|\phi| D=0
$$

and

$$
-i\left(-\partial_{r}-\frac{n+l+1}{r}\right) D-m|\phi| A=0
$$

which have small distances solutions,

$$
\left(\begin{array}{c}
A \\
D
\end{array}\right) \sim\left(\begin{array}{c}
r^{-n-l+|l|}+\ldots \\
r^{-n-l-1}+\ldots
\end{array}\right)
$$

and

$$
\left(\begin{array}{l}
A \\
D
\end{array}\right) \sim\left(\begin{array}{c}
r^{n}+\ldots \\
r^{n+|l|+1}+\ldots
\end{array}\right)
$$

Both of these solutions are regular for $n=0,1, . .,-l-1$; we have $|l|$ zero modes for $l$ negative. 
The presence of the GUT string becomes important in the region between the GUT length scale and the fermion's length scale. Here the GUT gauge field has attained its asymptotic form and the fermion mass term can still be neglected. The Dirac equations have a form similar to those above with $n$ and $l$ shifted by contributions to the $1 / r$ terms coming from the GUT gauge field. In this intermediate region some of the spinor components may vary as negative powers of $r[9]$. However, this behaviour does not persist into the origin, such zero modes are not destroyed but are simply 'amplified' at the origin.

Hence we have shown that, even taking the core of the GUT string into account, we have zero modes if $|\alpha / q|>1 / 2$. The existence of the zero modes results in the string becoming superconducting at the electroweak scale.

If the string does not wind there are no zero modes. However, the dip in the electroweak Higgs VEV constitutes a two-dimensional potential well. Two-dimensional wells are similar to potential wells in one-dimension and always have a bound state, unlike in three dimensions. Thus, there are always bound states in the Dirac spectrum.

Consider the ansatz above with $l=k=0$. The Dirac equation can then be written in the form

$$
-\gamma^{0}\left(\begin{array}{cccc}
0 & -i\left(\begin{array}{c}
\partial_{r}+\frac{n+1}{r} \\
-a Z_{\theta}+c X_{\theta}
\end{array}\right) & m|\phi| & 0 \\
-i\left(\begin{array}{c}
-\partial_{r}+\frac{n}{r} \\
-a Z_{\theta}+c X_{\theta}
\end{array}\right) & 0 & 0 & m|\phi| \\
-m|\phi| & 0 & 0 & -i\left(\begin{array}{c}
-\partial_{r}-\frac{n+1}{r} \\
+b Z_{\theta}-d X_{\theta}
\end{array}\right) \\
0 & -m|\phi| & -i\left(\begin{array}{c}
\partial_{r}-\frac{n}{r} \\
+b Z_{\theta}-d X_{\theta}
\end{array}\right) & 0
\end{array}\right)\left(\begin{array}{c}
A \\
B \\
C \\
D
\end{array}\right)=w\left(\begin{array}{c}
A \\
B \\
C \\
D
\end{array}\right)
$$

and we can identify the Hamiltonian operator,

$$
\hat{H}=\left(\begin{array}{cccc}
0 & i\left(\begin{array}{c}
\partial_{r}+\frac{n+1}{r} \\
-a Z_{\theta}+c X_{\theta}
\end{array}\right) & -m|\phi| & 0 \\
-i\left(\begin{array}{c}
-\partial_{r}+\frac{n}{r} \\
-a Z_{\theta}+c X_{\theta}
\end{array}\right) & 0 & 0 & m|\phi| \\
-m|\phi| & 0 & 0 & -i\left(\begin{array}{c}
-\partial_{r}-\frac{n+1}{r} \\
+b Z_{\theta}-d X_{\theta}
\end{array}\right) \\
0 & m|\phi| & i\left(\begin{array}{c}
\partial_{r}-\frac{n}{r} \\
+b Z_{\theta}-d X_{\theta}
\end{array}\right) & 0
\end{array}\right)
$$

If we can construct a state, $\psi$, such that $<\psi|\hat{H} \hat{H}| \psi>$ is less than $m^{2} \eta^{2}$ we can argue from the variational principle that there is a state somewhere between the positive and negative energy continua. Consider first the state

$$
\psi=\left(\begin{array}{c}
\zeta(r) \\
0 \\
0 \\
0
\end{array}\right),
$$

then

$$
\begin{aligned}
& <\psi|\hat{H} \hat{H}| \psi>=\int d^{2} x \zeta^{*}\left(m^{2}|\phi|^{2}+\left(\partial_{r}+\frac{n+1}{r}-a Z_{\theta}+c X_{\theta}\right)\left(-\partial_{r}+\frac{n}{r}-a Z_{\theta}+c X_{\theta}\right)\right) \zeta . \\
= & 2 \pi \int r d r\left(m^{2}|\phi|^{2}|\zeta|^{2}+\left|\left(-\partial_{r}+\frac{n}{r}-a Z_{\theta}+c X_{\theta}\right) \zeta\right|^{2}\right)+2 \pi\left[r \zeta^{*}\left(-\partial_{r}+\frac{n}{r}-a Z_{\theta}+c X_{\theta}\right) \zeta\right]_{0}^{\infty},
\end{aligned}
$$


after integrating by parts. Now consider the profile function $\zeta$. Let $\zeta_{0}$ satisfy

$$
\left(-\partial_{r}+\frac{n}{r}-a Z_{\theta}+c X_{\theta}\right) \zeta_{0}=0
$$

The solution is $\zeta_{0} \sim r^{n}$ at small $r$ and, using the asymptotic forms of the gauge fields, $\zeta_{0} \sim r^{[n-a(2 \alpha / G q)+c(1 / q)]}:=r^{s}$ at large $r$. For $n=0$ the small $r$ form is a constant and the large distance form is a decaying power law if the gauge field terms have the appropriate sign. We now introduce an exponential damping factor to ensure that the state is normalisable and consider $\zeta=\zeta_{0} \mathrm{e}^{-\lambda r}$.

If this state is normalisable with $\lambda=0$ then only the first term in (30) survives. The integral is less than the vacumm case when $|\phi|$ drops below its vacuum value. Alternatively, if a non-zero value of $\lambda$ is required to produce a normalisable state, we have extra contributions to the expectation value,

$$
<\psi|\hat{H} \hat{H}| \psi>=2 \pi \int r d r \mathrm{e}^{-2 \lambda r} \zeta_{0}\left(m^{2}|\phi|^{2}+\lambda^{2}\right) \zeta_{0}+2 \pi \lambda\left[\zeta_{0}^{2}\left(r \mathrm{e}^{-2 \lambda r}\right)\right]_{0}^{\infty}
$$

We are interested in small values of $\lambda$ and in this case the normalisation integral is dominated by the large distance contributions, giving a normalisation factor proportional to $\lambda^{1+s}$. The dip in the Higgs field occurs in the string core where the exponential factor can be ignored. Thus the only $\lambda$ dependence in the mass term comes from the normalisation factor and hence the energy reduction due to the dip in the Higgs profile varies as $\lambda^{2+2 s}$. The boundary term in the expectatioin value vanishes and the extra contribution varies as $\lambda^{2}$. If $s$ is negative, by making $\lambda$ suitably small we can produce a trial spinor whose squared energy lies below the continuum level.

Similar arguments can be constructed using a trial spinor with any one component nonzero. If the second component is taken to be nonzero, the order of the differential operators in (30) is reversed, the effective sign of the gauge field terms is reversed and this component will provide a decaying $\zeta_{0}$ if the first component did not.

We have shown that the string will have fermionic bound states if there is a dip in the scalar potential. These bound states can carry a current [6].

\section{5) Current Carrying Options}

We have constructed a composite string by considering couplings between the electroweak theory and a higher energy, string forming sector. We always find a layer of nontrivial electroweak fields surrounding the GUT string core. Depending on the ratio of gauge couplings, $|\alpha / q|$, the electroweak Higgs field can wind, leading to a stable electroweak string surrounding the GUT core. This gives rise to fermion zero modes and the string becomes superconducting at the electroweak scale. The current carried will be of the electroweak scale, this may be too small to produce dramatic cosmological effects, but could alter some of the cosmological predictions of the cosmic string scenario. This is under investigation.

For $|\alpha / q|<1 / 2$ the electroweak Higgs field does not wind, there are no fermion zero modes, but there are bound states which can carry a current. In this case the current 
is smaller than in the zero mode case and is not persistent, the size and dissipation time being determined by the mass of the bound state[6].

\section{Acknowledgements}

This work is supported in part by PPARC. We wish to thank M. Goodband and A. Yates for discussions.

\section{References}

1. M.B. Hindmarsh and T.W.B. Kibble, Rep Prog Phys, 58 (1995) 477; A. Vilenkin and E.P.S. Shellard, 'Cosmic Strings and Other Topological Defects' (Cambridge University Press) 1994

2. E. Witten, Nucl Phys 249 (1985) 557

3. A.C. Davis and P. Peter, Phys Lett 358 (1995) 197

4. R. Brandenberger, B. Carter, A.C. Davis and M. Trodden, hep-ph 9605382,

5. A. Everett, Phys Rev Lett 61 (1988) 1807; T.W.B. Kibble and A. Yates, private communication and in preparation; A. Yates, EU Conference, Capri (1996)

6. C.T. Hill and L.M. Widrow, Phys Lett B189 (1987) 17; M. Hindmarsh, Phys Lett B200 (1988) 429

7. R.L. Davis and E.P.S. Shellard, Nucl Phys B323 (1989) 209

8. W.B. Perkins and A.C. Davis, Nucl Phys B406 (1993) 377

9 M. Goodband and M. Hindmarsh, Phys Lett B (1996)

10. A. Achucarro, R. Gregory, J. Harvery and K. Kuijken, Phys Rev Lett 72 (1994) 3646

11. M.A. Earnshaw and W.B. Perkins, Phys Lett B328 (1994) 337

12. R. Jackiw and P. Rossi, Nucl Phys B190 (1981) 681 


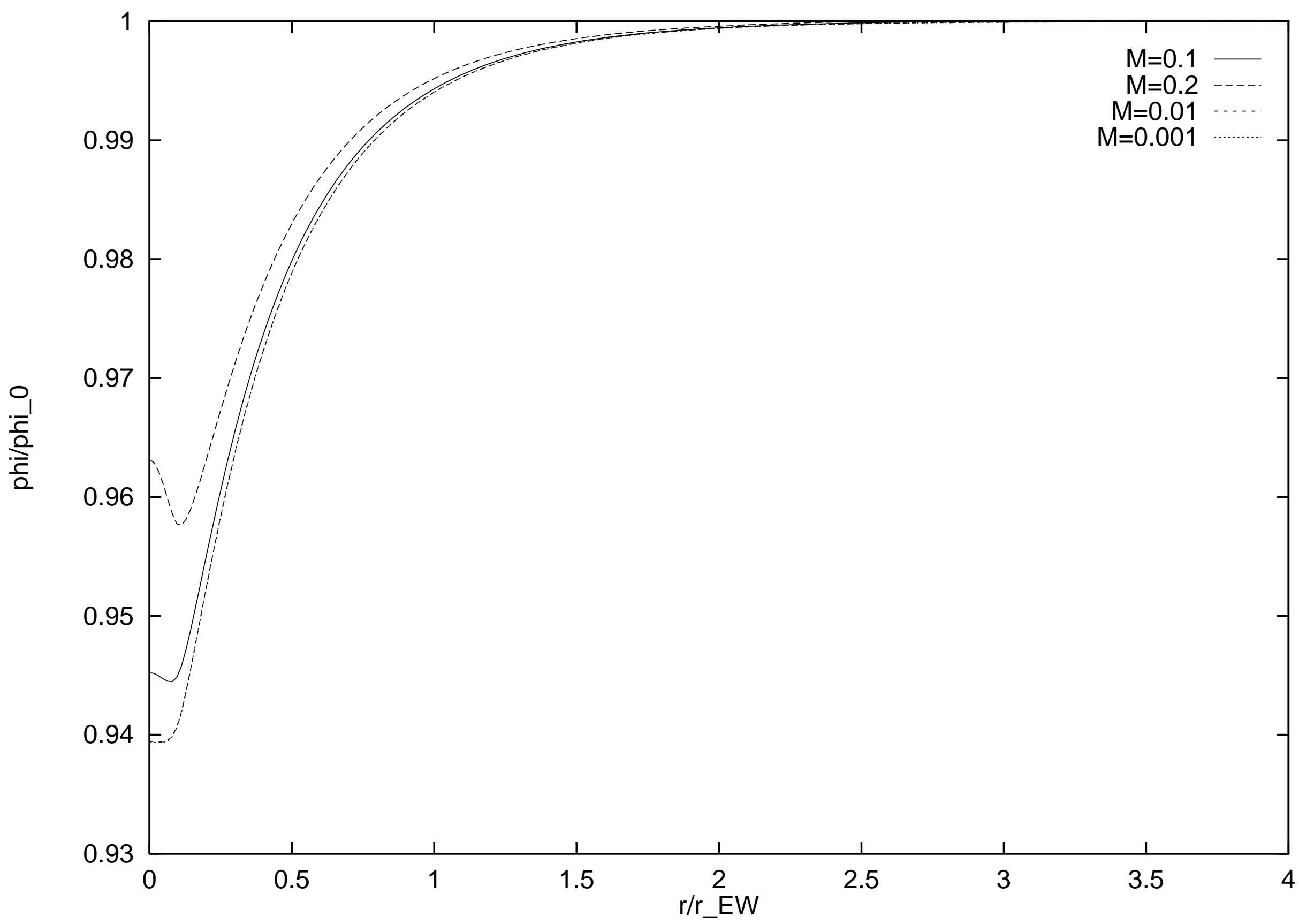

Fig.1. Higgs profile as a function of the scalar cross coupling, $M$ 


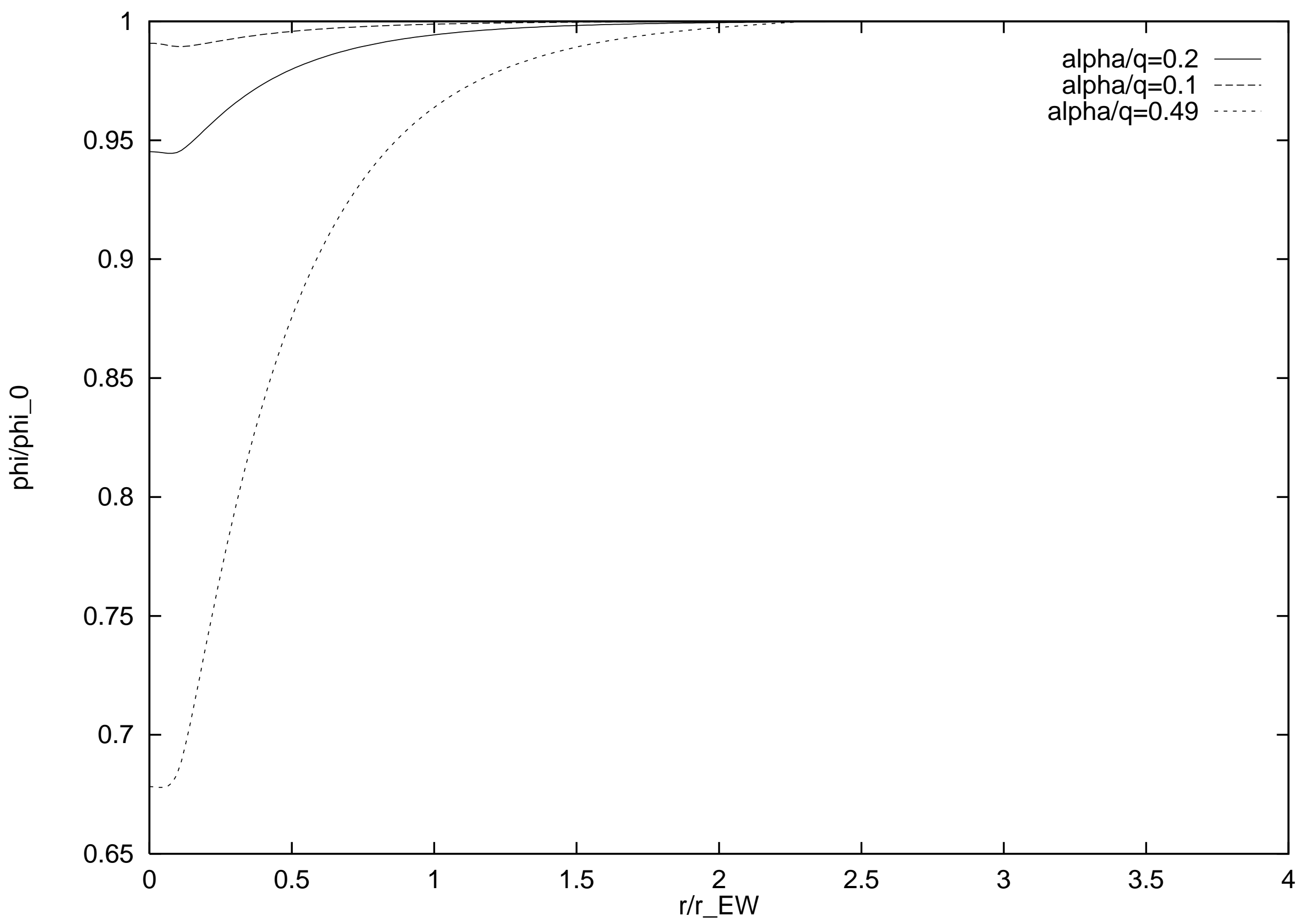

Fig.2. Higgs profiles as a function of gauge couplings ratio, alpha/q 


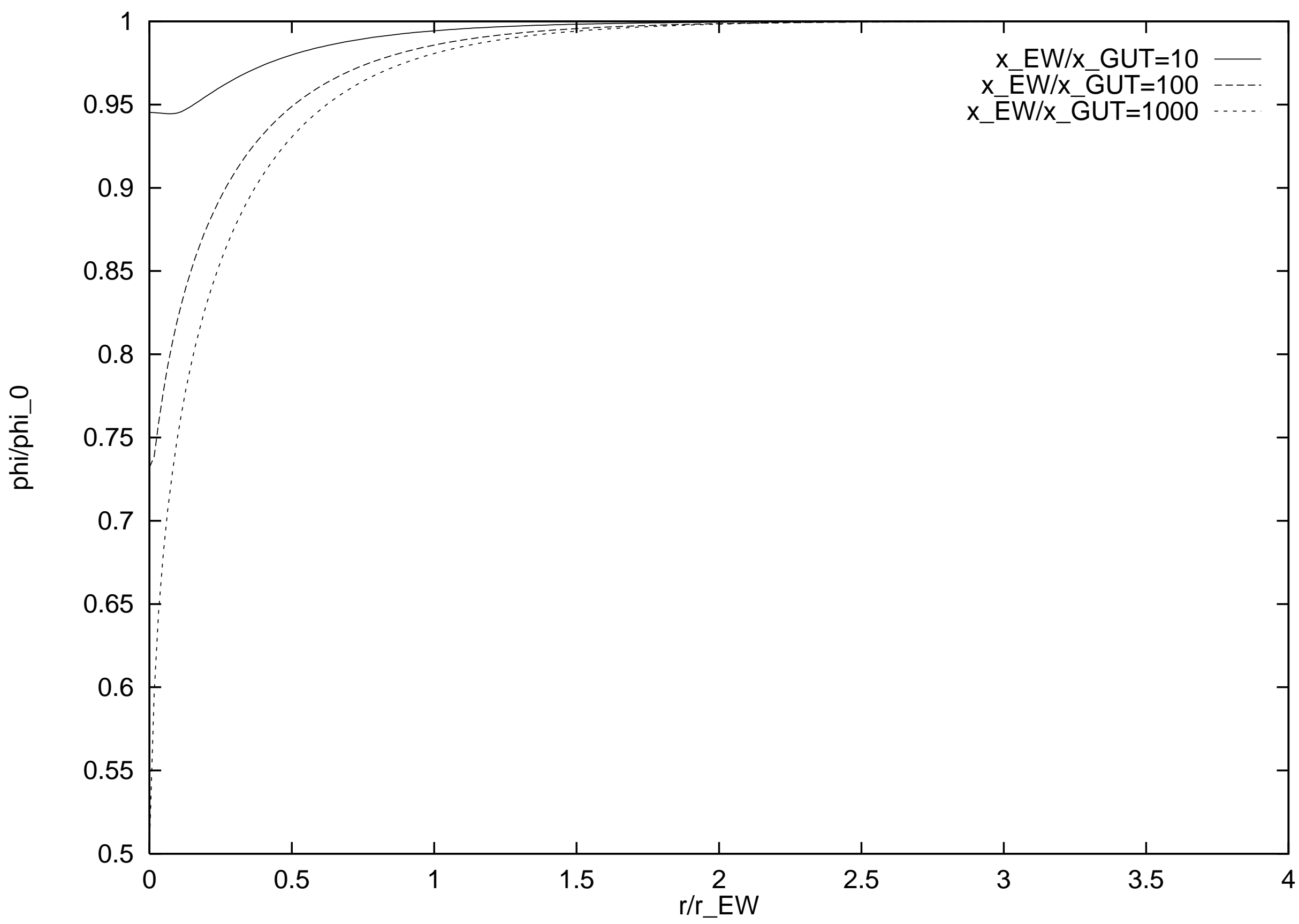

Fig.3. Higgs profiles for different ratios of the EW scale to the GUT scale 


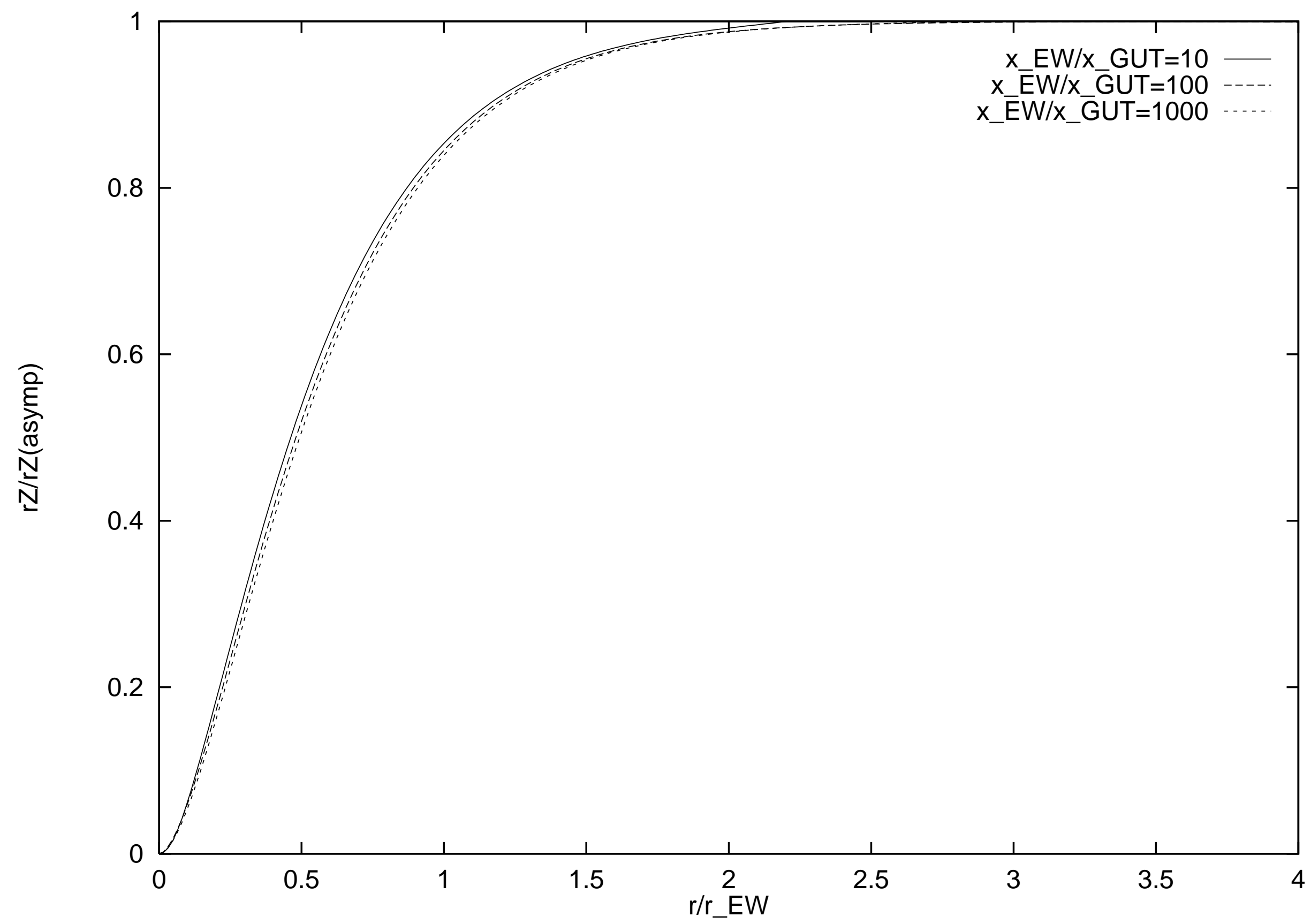

Fig.4. Gauge field profiles for different ratios of the EW scale to the GUT scale 


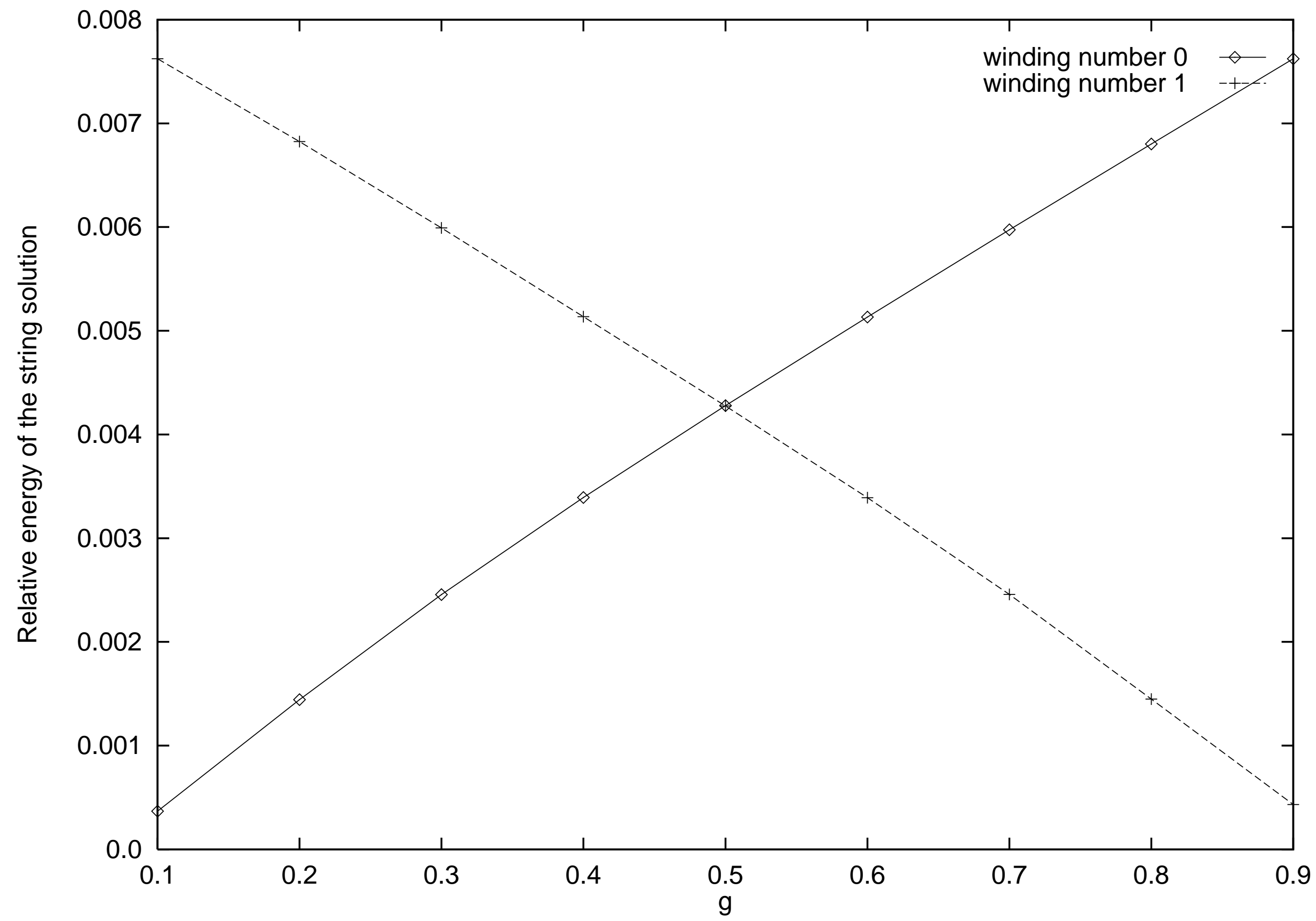

Fig.5. Energy of electroweak fields as a function of the effectivegauge coupling ratio for two winding numbers 